\title{
Battlefields of knowledge, Norman Long et Ann Long, London, 1992, 305 pp.
}

Ibrahim Saadou

\section{(2) OpenEdition \\ Journals}

Édition électronique

URL : http://journals.openedition.org/apad/2653

DOI : 10.4000/apad.2653

ISSN : 1950-6929

Éditeur

LIT Verlag

Édition imprimée

Date de publication : 1 décembre 1993

\section{Référence électronique}

Ibrahim Saadou, «Battlefields of knowledge, Norman Long et Ann Long, London, 1992, 305 pp. », Bulletin de l'APAD [En ligne], 6 | 1993, mis en ligne le 10 mars 2008, consulté le 21 septembre 2020 URL : http://journals.openedition.org/apad/2653; DOI : https://doi.org/10.4000/apad.2653

Ce document a été généré automatiquement le 21 septembre 2020.

Bulletin de I'APAD 


\title{
Battlefields of knowledge, Norman Long et Ann Long, London, 1992, 305 pp.
}

\author{
Ibrahim Saadou
}

1 Norman Long et son équipe ne donnent pas moins à leur ouvrage que le titre de "'Champs de bataille des savoirs". Le livre collectif produit par Norman Long et son équipe est le fruit de leurs expériences de terrain en Amérique Latine et en Afrique et de leurs discussions théoriques. Il s'inscrit sur le terrain du développement et du changement social, où le chercheur est lui-même un acteur impliqué dans ce qu'il étudie. Le processus de production de la connaissance est dialogique voire dialectique entre le chercheur et son objet d'étude. Il n'y a donc pas d'observation "'objective", "neutre" et "'détachée".

Sans sous-estimer l'importance du "macro", les auteurs ont volontairement privilégié "les petites choses" et focalisé l'attention sur certains aspects négligés de la vie quotidienne. L'approche initiée par Long et son équipe est orientée sur l'acteur et basée sur la compréhension de la vie quotidienne. Ses concepts analytiques sont ceux d'acteur social, de monde vécu ou d'univers de vie, de construction de savoirs, d'action stratégique, d'enrôlement. de marges de manœuvre, d'entremêlement des projets, de pratiques discursives, de réalités multiples, d'arènes de luttes où s'affrontent valeurs et pouvoirs à travers des structures intermédiaires et différenciées, des champs organisationnels et des réseaux de savoirs. Cette approche se veut non linéaire et non déterministe : en effet, des circonstances ou des interventions structurelles similaires peuvent produire des réponses ou des résultats différents selon les contextes, en fonction de mélanges compliqués de conséquences prévisibles et imprévisibles de l'action humaine. Le processus de production des savoirs doit être saisi comme "discontinuité", "transformation" émergeant comme un produit de l'interaction et du dialogue entre acteurs spécifiques.

3 Si pendant les années 1970 et 1980 le modèle néo-marxiste a permis d'expliquer certains aspects des problèmes de développement du Tiers-Monde, il a méconnu les 
capacités des populations et groupes locaux. L'œuvre est aussi une critique des techniques dites "d'évaluation rapide", "méthode sondéo", "ethnographie rapide", "recherche et évaluation participative", sans oublier également toutes méthodes qui se soucient peu de la théorisation. C'est aussi une critique de l'individualisme méthodologique et du structuralisme.

Le livre est constitué de quatre parties. La première, (l'introduction), constitue un excellent résumé de l'œuvre. La deuxième partie traite de la crise dans laquelle se trouve la sociologie du développement après l'effondrement des grandes orthodoxies aussi bien libérales que radicales et montre la naissance de l'approche "centrée sur l'acteur" (actor-oriented), où l'on analyse comment les acteurs sociaux interprètent de nouveaux éléments dans leurs "univers de vie" (life worlds) et comment les savoirs sont les produits de négociations au cours de types variés d'interactions sociales.

Pieter de Vriès, à partir de son expérience sur des projets de développement au Costa Rica, décrit comment le processus de recherche est un enrôlement mutuel entre le chercheur et le "recherché", processus qui ne s'arrête d'ailleurs pas sur le terrain mais continue même pendant l'interprétation du matériel de terrain. Torres montre comment l'intervention sociologique n'est pas seulement sujette à l'essai et à l'erreur, mais est négociée et construite pendant le processus de recherche.

6 Dans la troisième partie. Andrew Long explore la construction sociale de la connaissance dans la vie quotidienne autour de situations variées. L'auteur se réfère à ses expériences chez les agriculteurs Zambiens. Gérard Verschoor, partant de son expérience chez de petits entrepreneurs Mexicains, argumente pour une approche qui donne plus de poids à la façon dont les acteurs locaux "internalisent" leurs expériences d'évaluation, à partir de leurs savoirs et de leurs valeurs culturelles, et en fonction d'une organisation stratégique des réseaux sociaux et des relations spatiales. Ces petits entrepreneurs développent des représentations du monde social dans lequel ils opèrent, représentations qu'ils utilisent pour structurer les engagements et contributions des partenaires et clients de leur business.

7 Toujours au Mexique, Monique Nuijeen prenant le contre-pied de toute une littérature qui proclame le succès de "la modernisation occidentale", montre que cette vision est très éloignée de la réalité. Elle met l'accent sur l'importance du savoir local et des systèmes culturels, et fait ressortir tous les types de réseaux effectifs et groupes d'alliance qui lient ensemble fermiers, entrepreneurs et agents de l'Etat.

8 Dans la quatrième partie, Norman Long et Alberto Arce, partant de leurs terrains mexicains, mettent l'accent sur l'étude des processus d'intervention, notamment les rapports État-paysans. L'échec d'un "technicisme" à faire accepter aux paysans une nouvelle variété de maïs, traduit tout le problème des incompatibilités et des pouvoirs différentiels entre les deux mondes et leurs formes de la connaissance quotidienne. Magdalena Villarred s'interroge, elle, sur l'utilité des activités de développement. En comparant deux groupes de femmes, dont l'un crée sous les auspices d'un programme gouvernemental et l'autre informel, elle arrive à la conclusion que les initiatives d'intervention "pour renforcer le faible", débouchent sur une contradiction. Cela pose tout le problème des interventions volontaristes.

9 Ce bref résumé n'épuise pas la remarquable œuvre de Norman Long et son équipe. Il faut aller au texte lui-même pour découvrir toute son originalité et sa richesse. 


\section{AUTEUR}

IBRAHIM SAADOU

École des Hautes Études en Sciences Sociales. Marseille 